\title{
Incomplete descriptions and relevant entropies Roger Balian
}

\author{
Service de Physique Théorique, CEA/Saclay \\ F-91191 Gif-sur-Yvette Cedex, France
}

\begin{abstract}
Statistical mechanics relies on the complete though probabilistic description of a system in terms of all the microscopic variables. Its object is to derive therefrom static and dynamic properties involving some reduced set of variables. The elimination of the irrelevant variables is guided by the maximum entropy criterion, which produces the probability law carrying the least amount of information compatible with the relevant variables. This defines relevant entropies which measure the missing information (the disorder) associated with the sole variables retained in an incomplete description. Relevant entropies depend not only on the state of the system but also on the coarseness of its reduced description. Their use sheds light on questions such as the Second Law, both in equilibrium an in irreversible thermodynamics, the projection method of statistical mechanics, Boltzmann's $H$-theorem or spin-echo experiment.
\end{abstract}

\section{Introduction}

The concept of entropy underlies both thermodynamics and statistical physics, but its subtleties and its multiple aspects make it difficult to grasp. Our initial motivation is to understand how the entropy of thermodynamics $S_{\text {th }}$ is related to the entropy of statistical mechanics. The solution of this problem will turn out to require the introduction in statistical physics of several different entropies, which will enlighten some other questions.

For systems at equilibrium, the situation is simple, and it is dealt with in many tutorial books. At the macroscopic scale, the state of a system at equilibrium is characterized by some set of thermostatic variables, such as the particle number, the energy and the volume for a fluid. (We distinguish the thermodynamics of equilibrium from the thermodynamics of irreversible processes by terming them as "thermostatics" and "thermodynamics" proper, respectively.) The entropy $S_{\text {th }}$ is some function of these variables, a function that can be determined indirectly by experiments. At the microscopic scale, the same state is described by a density operator $D$ having the Boltzmann-Gibbs exponential form. The von Neumann entropy associated to $D$, defined by

$$
S(D) \equiv-k \operatorname{Tr} D \ln D,
$$

is then identified with the thermostatic entropy $S_{\text {th }}$; the Boltzmann constant $k=1.38 \times 10^{-23} \mathrm{JK}^{-1}$ entering (1.1) accounts for the conventional choice of 
the kelvin as the unit of temperature in thermal physics. This identification is justified through derivation of the Laws of thermostatics - in particular the Second Law - from the Boltzmann-Gibbs distributions of statistical physics. Moreover the microscopic approach allows us in principle to evaluate $S_{\text {th }}$, which macroscopically is an empirical quantity.

The identification of the entropy $S_{\text {th }}$ of thermodynamics with the von Neumann entropy $S(D)$ cannot, however, be extended to dynamical processes. Consider an isolated system off equilibrium. On the one hand, according to the Second Law, its thermodynamic entropy $S_{\text {th }}$ is larger in its final equilibrium state than in its initial state. If its evolution proceeds through states close to equilibrium, the Clausius-Duhem inequality expresses moreover that the time-derivative of $S_{\text {th }}$ is non-negative. On the other hand, the microscopic evolution is governed (in the Schrödinger picture) by the Liouville-von Neumann equation for the density operator,

$$
\mathrm{i} \hbar \frac{d D}{d t}=[H, D],
$$

where $H$ is the hamiltonian operator of the system. It follows from (1.1) and (1.2) that $S(D)$ does not vary with time. This constancy, when compared to the Second Law, constitutes a modern form of the paradox of irreversibility (W. Thomson 1874, J. Loschmidt 1876), which expresses a qualitative difference between microscopic and macroscopic dynamics. The solution of this paradox requires of course the thermodynamic entropy $S_{\text {th }}$ of non-equilibrium states to differ from the von Neumann entropy $S(D)$.

Actually, we shall associate below ( $\S 6$ and $\S 7$ ) various so-called relevant entropies with a given density operator $D$, and shall show $(\S 10)$ that one of them can be identified with $S_{\text {th }}$. We shall more generally illustrate the utility of the idea of relevant entropy, by showing how it underlies the projection method ( $\S 8$ and §9) and how it enlightens some questions of irreversibility in statistical physics ( $\S 11$ and $\S 12$ ). As a preliminary step, we briefly review ( $\S \S 2$ to 5 ) some aspects of the concepts of state and of entropy in statistical physics, on which we shall rely in the following. In particular it will be important to distinguish the complete statistical description of a state $(\S 2)$ from its incomplete descriptions $(\S 5)$ and to grasp the meaning of the maximum entropy criterion $(\S 4)$. Although this article is self-contained, it is somewhat sketchy. Further explanations, details and developments can be found in the first few and last few chapters of reference [1]. 


\section{Complete statistical description: the density operator}

The state of a system at a given time can be defined in several ways that should not be confused.

(i) Thermodynamics relies on the identification of some set of macroscopic quantities $\mathcal{A}_{i}$, the control of which is sufficient to govern the whole macroscopic behaviour of the system. A state of this system is then characterized by specifying the quantities $\mathcal{A}_{i}$, irrespective of the microscopic variables which otherwise remain arbitrary. At the microscopic level, the thermodynamic description is thus incomplete.

(ii) At the other extreme, one can imagine states, termed as "microstates", in which all the variables are defined inasmuch as possible. In classical mechanics, when the uncertainly principle may be disregarded, nothing prevents such a definition of the variables to be perfect: a microstate is characterized by specifying the positions and momenta of all the particles (within permutations in the labels of indistinguishable particles). In quantum mechanics, a microstate must retain probabilistic features; it is represented as a ket, a vector in the Hilbert space associated with the system.

(iii) For large systems it is however impossible in practice to prepare a microstate. It is thus necessary to resort to statistics. The definition of a state therefore refers, not to a single system, but to a typical system chosen out of a statistical ensemble of systems all prepared under similar conditions. We recall in the continuation of this section the main features of this last type of state.

The quantum physical quantities, or "observables", are represented as hermitean operators $A$ which act in the Hilbert space of the system. In the classical limit, an observable tends to a random variable which is a function of the (random) positions and momenta of the particles. Thus, in quantum mechanics, the observables play the rôle of non-commutative random variables.

We characterize statistically a state by its density operator at the considered time. This means that we can evaluate the expectation value $\langle A\rangle$ of any observable $A$ at that time by means of the equation

$$
\langle A\rangle=\operatorname{Tr} A D
$$

The description of the system furnished by its density operator $D$ is thus statistical, since in general the variance $\left\langle A^{2}\right\rangle-\langle A\rangle^{2}$ does not vanish, and complete, since any expectation value can be derived from (2.1).

The density operator $D$ is therefore the tool which implements the correspondence $A \mapsto\langle A\rangle$ from the whole set of observables $A$ to their expectation values $\langle A\rangle$. Actually, in classical statistical mechanics, $D$ is replaced by an ordinary 
probability distribution, from which the expectation value of any observable results through an integration which replaces the trace of (2.1). Thus a density operator should be regarded as a probability distribution for non-commuting random variables. Conversely, a linear correspondence $A \mapsto\langle A\rangle$ defines a density operator $D$. The standard properties of $D$, normalization, hermiticity and positivity, express that the expectation value of the unit operator $I$ is $\langle I\rangle=1$, and that $\langle A\rangle$ is real, $\left\langle A^{2}\right\rangle$ is positive for any hermitean $A$.

A density operator $D$ is usually represented as a matrix $D_{\alpha \beta}$ in the Hilbert space. However, it may be convenient (and it will be necessary in $\S 8$ ) to regard the two indices $\alpha, \beta$ of this density matrix as a single, compound index. The density operator is then represented, no longer as a matrix but as a vector in the so-called Liouville space, where the compound index $\alpha, \beta$ plays the rôle of a single set of coordinates. We can then perform linear transformations on the compound index. This defines Liouville representations of quantum statistical mechanics, which encompass the standard matrix representations but are more general and more flexible [2][3][4][5]. In a Liouville representation, the observables are also represented as vectors with a compound index. They belong to the dual Liouville space, and an expectation value (2.1), written as

$$
\langle A\rangle=\sum_{\alpha \beta} A_{\alpha \beta} D_{\beta \alpha}=(A ; D),
$$

appears as a scalar product between the two vectors $A$ and $D$ of the two conjugate Liouville spaces. In a Liouville representation, the Liouville-von Neumann equation, which governs the evolution of $D$, takes the form

$$
\frac{d D}{d t}=\mathrm{L} D
$$

which is equivalent to (1.2). The liouvillian $L$ is a superoperator, represented in the Liouville space as a matrix. In the Hilbert space, it appears as a tensor with $2 \times 2$ indices, and (2.3) is written in terms of the density matrix $D_{\alpha \beta}$ as

$$
\frac{d D_{\alpha \beta}}{d t}=\sum_{\gamma \beta} \mathrm{L}_{\alpha \beta, \gamma \delta} D_{\delta \gamma} ;
$$

comparison with the more standard notation (1.2) shows that

$$
\mathrm{L}_{\alpha \beta, \gamma \delta}=\frac{1}{\mathrm{i} \hbar}\left(H_{\alpha \delta} \delta_{\beta \gamma}-H_{\gamma \beta} \delta_{\alpha \delta}\right)
$$

The simplest example of Liouville representation concerns the statistical state of a spin $\frac{1}{2}$. Rather than describing it by a $2 \times 2$ hermitean density matrix with 
trace 1, it is handy to represent it by a vector in a 3-dimensional Liouville space, with merely the expectation values of the Pauli matrices as components. The equation of motion (2.3) then describes directly the Larmor precession of this vector in a plane perpendicular to the magnetic field.

Another example of Liouville representation, the Wigner representation, is useful in the study of the classical limit of quantum statistical mechanics. In this limit, the observables tend to commuting random variables. For a system of $N$ indistinguishable particles, the density operator $D$ in the Wigner representation tends to an ordinary probability distribution in the $6 \mathrm{~N}$-dimensional phase space of coordinates and momenta of these particles; it can be identified with the probability density in phase space of classical statistical mechanics. The traces in (1.1) and in (2.1) or equivalently the scalar product (2.2) reduce to integrals in phase space, with the measure

$$
\prod_{i=1}^{N} d^{3} \mathbf{r}_{i} d^{3} \mathbf{p}_{i} / N ! h^{3 N}
$$

which arises from the summation over $\alpha$ and $\beta$ of (2.2). The equation of a motion (2.3) directly yields the classical Liouville equation.

\section{The statistical entropy as missing information}

Information theory associates with an event $n$, which has the probability $p_{n}$ to occur, its surprisal $I_{n}=-k \ln p_{n}$, a number which measures the amount of information that we gain when we get to know the occurrence of this event. Within the multiplicative constant $k$ which defines the unit of information, this logarithmic expression for $I_{n}$ is imposed by the condition that the information is additive when knowledge is gained by steps. The surprisal vanishes when the occurrence of $n$ is certain, it increases as its probability becomes weaker. We shall let $k=1$ in the continuation. This will imply that Boltzmann's constant is replaced by 1 , so that we take the joule as the unit of temperature.

For a set of exclusive events $n$, one of which is expected to occur, the statistical entropy is then defined as the average amount of information which is gained when either event occurs, that is,

$$
S\left(\left\{p_{n}\right\}\right)=\sum_{n} p_{n} I_{n}=-\sum_{n} p_{n} \ln p_{n}
$$

This expression, a function of the probabilities $p_{n}$, equivalently measures the amount of information which is missing when only the probabilities $p_{n}$ of occurrence of the various events $n$ are known. 
Returning to statistical mechanics, the eigenvalues $p_{n}$ of a density operator $D$ may be interpreted as ordinary probabilities of the microstates which are the corresponding eigenvectors of $D$ and which behave as exclusive events. The expression (3.1), rewritten as

$$
S(D)=-\operatorname{Tr} D \ln D
$$

is identified with the von Neumann entropy (1.1). It is thus interpreted as the lack of information which arises from the incompleteness of our statistical description by means of the density operator $D$. Like the density operator itself, the von Neumann entropy is a statistical quantity, since it is a natural measure of uncertainty. As such, it is not a property of the object at hand, but has partly a subjective nature linked to our knowledge of this object, which is extracted from a statistical ensemble.

This subjective character is somewhat hidden in the alternative interpretation of $S(D)$ as a measure of disorder. We can argue, however, that "disorder" and "missing information" are synonymous. Consider, for instance, the 13 spades taken from a pack of cards. A first configuration, in which they are set according to the decreasing values of bridge, ace, king, ..., two, displays perfect order; its statistical entropy vanishes. Some second configuration, reached after a long shuffling, appears completely disordered. Nothing allows us to recognize any special feature in it. The reason for this difference is that we regard the former configuration as unique (with probability 1), while we regard the latter as just one among all 13! possible configurations. Before looking at it, we assign to it the probability of occurrence $1 / 13$ !, and the entropy is therefore $\ln 13$ !. However, if the shuffling is performed by a skilful conjurer who controls every card, he knows perfectly the arrangement in the second configuration, which for him is perfectly ordered. Indeed he can reshuffle it back to the first configuration.

A similar situation occurs in spin-echo experiments. At the initial time, the spins are prepared in a perfectly ordered configuration: they all point in the $x$-direction. A permanent magnetic field is applied in the $z$-direction, but its magnitude is not quite the same at the site of each spin, so that the Larmor frequencies of the various spins are slightly dispersed around their average value. From the time 0 on, the spins precess rapidly in the $x y$-plane, but at slightly different speeds, so that the total magnetization $M$ decreases in length while rotating at the average Larmor frequency: it relaxes to zero. We thus reach after some time $T$ a completely disordered configuration where the spins point towards arbitrary directions of the $x y$-plane. However, this configuration is not disordered for the spin-echo experimentalist, who knows where it was issued from. He takes advantage of this knowledge by applying to the system at the time $T$ a brief pulse of magnetic field along $x$, which suddenly rotates each spin by $\pi$, replacing it by its symmetric with respect to the $x$-axis. Thus, the spins which were ahead 
in the precession are now behind and conversely. After a second delay $T$, the precession, which again takes place at different speeds according to the site of each spin, produces back the original ordered configuration. We shall return in $\S 12$ to this example which suggests that order, or entropy, is not defined uniquely in the seemingly disordered configuration attained after relaxation.

\section{The maximum entropy criterion}

Owing to its interpretation as the missing amount of information associated with the probability distribution $p_{n}$, the statistical entropy (3.1) is currently used as a tool for statistical inference. The purpose of statistical inference is to make reasonable predictions about some quantities, starting from an exact or a statistical knowledge of some other quantities. We thus have to assign to the considered set of elementary events $n$ a probability distribution $p_{n}$, which should of course account for the available data but should otherwise be unbiased.

It is natural to admit that, among two probability laws, the more biased one is the one which carries more information. This assumption leads us to assign the probabilities $p_{n}$ of each event by relying on the maximum entropy criterion, as was first advocated by Jaynes [6]. The knowledge of the available data, whether they are specified exactly or statistically, first sets up constraints on the probabilities $p_{n}$. Then, among all probability laws compatible with these constraints, we select the one for which the statistical entropy (3.1) is largest. Any other probability law compatible with the data would have a smaller statistical entropy, i.e., would carry more information than the minimum amount needed to account for these data. Choosing probabilities by means of the maximum entropy criterion thus amounts to retain all the available information while discarding any other irrelevant information.

This method has successfully been applied to numerous and varied problems, ranging from signal theory to image processing and from detection of astrophysical objects to determination of protein structures. Its extension to statistical mechanics relies on the maximization of the von Neumann entropy (3.2), a function of $D$ which measures the lack of information. A density operator is thereby assigned to a state which is characterized by some given conditions, by looking for the maximum of $S(D)$ under constraints that account for these conditions. We shall work out this procedure in $\S 6$ and $\S 7$ after having shown ( $\$ 5)$ some examples of such constraints. A direct justification of the maximum entropy criterion in statistical mechanics, based on Laplace's indifference principle (equal probabilities should be assigned when nothing in known) rather than on the existence of von Neumann's entropy, is given in ref. [7]. 


\section{Relevant observables and incomplete descrip- tions}

We have noted $(\S 2, \mathrm{i})$ that the state of a system is characterized at the macroscopic level by some partial set of quantities $\mathcal{A}_{i}$. When only such an information is available, the microscopic description of the state is incomplete, since we know nothing about the other microscopic variables. We shall identify a macroscopic quantity $\mathcal{A}_{i}$ with the expectation value $\left\langle A_{i}\right\rangle$ of the corresponding observable $A_{i}$. We thus deal with a system for which only the expectation values $\left\langle A_{i}\right\rangle$ of some set of observables $A_{i}$, that we refer to as the relevant set $R \equiv\left\{A_{i}\right\}$, are specified. Such a partial knowledge, both statistical and incomplete, is not sufficient to characterize the density operator $D$, which is merely constrained to satisfy the set of equations

$$
\operatorname{Tr} D A_{i}=\left\langle A_{i}\right\rangle=\mathcal{A}_{i}
$$

Note that for some other quantities the correspondence from macrophysics to microphysics is more straightforward. For instance, if the particle number of a system is specified exactly, leaving no room for any statistical fluctuation, this particle number enters the microscopic description through the very definition of the Hilbert space of the system. As another example, the volume $\Omega$ of a fluid appears in the microscopic theory implicitly through the hamiltonian. Such quantities are accounted for directly in the microscopic theory, and not as expectation values (5.1) of observables.

Let us illustrate the above correspondence between macroscopic quantities $\mathcal{A}_{i}$ and relevant observables $A_{i}$ with a few examples.

In thermostatics, the variables $\mathcal{A}_{i}$ are those which characterize the macroscopic state in equilibrium. For instance the thermostatic state of a simple fluid, made of a single type of identical molecules enclosed in a vessel with volume $\Omega$, is characterized by two macroscopic data $\mathcal{A}_{i}$, namely its particle number $\mathcal{N}$ and its internal energy $\mathcal{U}$. We wish to describe this system at the microscopic level in terms of a density operator $D$ in the Fock space, that is, in the Hilbert space with an arbitrary number of particles. Our first datum is then the expectation value $\langle N\rangle=\mathcal{N}$ of the particle number operator (in the Fock space), while the internal energy $\mathcal{U}$ is identified with the expectation value $\langle H\rangle$ of the hamiltonian operator $H$. The set $R$ of relevant observables contains here two elements, the operators $N$ and $H$. We noted above that the volume $\Omega$ enters the problem through the expression of $H$, which contains a potential confining the particles within $\Omega$.

Our identification of macroscopic data $\mathcal{A}_{i}$ with microscopic expectation values $\left\langle A_{i}\right\rangle$ raises a question, since the microscopic quantities, particle number and energy, are allowed to fluctuate freely around their expectation values, in contrast to their macroscopic counterparts. However, the statistical fluctuations which 
appear in statistical mechanics for macroscopic systems turn out to be extremely small (of order $\langle N\rangle^{-1 / 2}$ in relative value); they are actually much smaller than the experimental uncertainties on the variables $\mathcal{A}_{i}$, so that this qualitative discrepancy between the macroscopic and the microscopic descriptions is ineffective.

For other systems in thermostatic equilibrium, the set $R$ of relevant observables $A_{i}$ can involve, apart from $N$ and $H$, additional constants of the motion, represented by observables which commute with $H$. When some invariance is broken, we have moreover to include order parameters, which also keep a fixed value in time at least on the time-scales of observation.

For thermodynamic processes close to equilibrium, a state is described macroscopically at each time by distinguishing within the system a set of subsystems $a$, each of which is nearly in local equilibrium [8]. The macroscopic state is then characterized by specifying the thermostatic variables for each subsystem. For instance, in the thermodynamic or hydrodynamic regime, a simple fluid can be analyzed into volume elements, larger than the mean free path, so as to have reached local equilibrium, but sufficiently small so as to be practically homogeneous. The variables $\mathcal{A}_{i a}$ are then the number of particles in each volume element, their total energy and their total momentum (or equivalently the local densities of particles, energy and momentum, smoothed over the mean free path); the compound index $i a$ denotes here both the location $a$ in space and the nature $i$ of the physical quantity. The relevant set $R$ of observables $A_{i a}$ are the corresponding operators (or random variables in the classical limit).

A similar analysis holds quite generally, for thermal, mechanical, chemical, electrical or magnetic processes, provided they are sufficiently slow so as to ensure local equilibrium. The thermodynamic quantities are most often the locally conserved quantities, but may also include other variables varying slowly on the microscopic scale.

For more rapid processes, these thermodynamic variables are not sufficient to determine the physics of the system; more detailed quantities should be included into the set $\mathcal{A}_{i}$, although many microscopic variables are still kept aside. For instance, the description of a gas suited for the ballistic regime, or Boltzmann description, requires an analysis, not only in the ordinary space, but in the 6dimensional single-particle phase space. The set of relevant variables $\mathcal{A}_{i}$ are then the densities $f(\mathbf{r}, \mathbf{p})$ of particles at each point of this space; the index $i$ stands here for $(\mathbf{r}, \mathbf{p})$. In the Boltzmann description, all the 2-point, 3-point, ... correlations are disregarded. At the microscopic level, the set $R$ of relevant observables includes all the single-particle observables, but only these. The components of the density operator (or, in the classical limit, of the probability density in the $6 \mathrm{~N}$ dimensional phase space) referring to 2-particle, 3-particle... observables remain unspecified.

Other examples of incomplete descriptions are provided, on the one hand by coarse-graining, on the other hand by collective variables. Consider for instance large nuclei. It is impossible to describe their state fully, but their dynamics can 
be accounted for by following only some set of collective variables, such as the ones which describe the shape of the nucleus in a fission problem.

The set of relevant observables always includes the quantities which can experimentally be observed or controlled. However, the above examples show that for dynamical problems our description also includes other quantities, needed to account theoretically for the physics of the system. For instance, in fluid dynamics, the densities of particles, energy and momentum are not controlled everywhere, but they must be introduced to write the basic, Navier-Stokes and Fourier, equations.

\section{$6 \quad$ Least biased states and relevant entropies}

We return to inference in statistical mechanics, first focusing on the following single-time problem. Let $R \equiv\left\{A_{i}\right\}$ be some set of relevant observables. We assume that their expectation values $\left\langle A_{i}\right\rangle=\mathcal{A}_{i}$ are specified at some given time, but that nothing else is known. From this incomplete information, we wish to make a reasonable statistical guess about the expectation value $\langle B\rangle$ of any other observable $B$ at the considered time. Equivalently, we wish to construct at that time the density operator $D$ which encompasses our whole statistical knowledge ( $\S 2)$, both within and outside $R$.

To this aim, we use the maximum entropy criterion of $\S 4$. The density operator $D_{0}$ to be assigned to the system should both fulfil the constraints (5.1) expressing that $\left\langle A_{i}\right\rangle=\mathcal{A}_{i}$ and render the von Neumann entropy (3.2) maximum, so as to avoid including in $D$ any additional information. The construction of the least biased state $D_{0}$ is therefrom achieved through introduction of a lagrangian multiplier $\gamma_{i}$ associated with each constraint (5.1). The result has the form

$$
D_{0}=\exp \left[-\Psi-\sum_{i} \gamma_{i} A_{i}\right],
$$

where the term $\Psi$ accounts for the normalization $\operatorname{Tr} D_{0}=1$ of $D_{0}$. The exponential in (6.1) reflects the occurrence of a logarithm in $S(D)$.

A direct proof of (6.1) relies on the inequality

$$
S(D)<-\operatorname{Tr} D \ln D^{\prime}
$$

which holds for any pair of density operators $D$ and $D^{\prime}$. If in (6.2) we replace $D^{\prime}$ by (6.1) and $D$ by any other density operator satisfying the constraints (5.1), the right-hand side reads

$$
-\operatorname{Tr} D \ln D_{0}=\Psi+\sum_{i} \gamma_{i}\left\langle A_{i}\right\rangle=-\operatorname{Tr} D_{0} \ln D_{0}=S\left(D_{0}\right)
$$


and we readily see that $S\left(D_{0}\right)>S(D)$, as we anticipated.

The determination of $D_{0}$ is achieved by specifying the values of the parameters $\Psi$ and $\gamma_{i}$. The normalization condition, written as

$$
\Psi\left(\left\{\gamma_{i}\right\}\right)=\ln \operatorname{Tr} \exp \left[-\sum_{i} \gamma_{i} A_{i}\right]
$$

first defines $\Psi$ as a function of the multipliers $\gamma_{i}$. The latter quantities are then determined by inserting (6.1) into (5.1), which yields a set of equations,

$$
-\frac{\partial \Psi}{\partial \gamma_{i}}=\mathcal{A}_{i}
$$

relating the multipliers $\gamma_{i}$ and the macroscopic data $\left\langle A_{i}\right\rangle=\mathcal{A}_{i}$.

The above procedure is standard in thermostatics. If for instance the relevant set $\left\{A_{i}\right\}$ consists of the particle number operator $N$ and the hamiltonian $H$, we find thus a statistical justification for the grand canonical density operator (6.1). The data $\mathcal{A}_{1}$ and $\mathcal{A}_{2}$ are $\mathcal{N}$ and $\mathcal{U}$, respectively, while the multipliers $\gamma_{1}$ and $\gamma_{2}$ are identified with $-\mu / k T$ and $1 / k T$, respectively, where $T$ is the temperature (in kelvin) and $\mu$ is the chemical potential. The function (6.4) is then identified with the Massieu thermodynamic potential, and (6.3) with the usual entropy of thermostatics since (6.5) implies the same equation

$$
d S=\gamma_{1} d \mathcal{N}+\gamma_{2} d \mathcal{U}=\frac{1}{T}(d \mathcal{U}-\mu d \mathcal{N})
$$

as in macroscopic physics.

A similar formal structure, analogous to that of thermostatics, occurs for any imperfectly known state at a given time, when the expectation values $\left\langle A_{i}\right\rangle=\mathcal{A}_{i}$ of some relevant set $R=\left\{A_{i}\right\}$ of observables (as exemplified in $\S 5$ ) are the only available information. A multiplier $\gamma_{i}$ is associated with each given quantity $\mathcal{A}_{i}$. The least biased state $D_{0}$ (eq. 6.1) is characterized equivalently by the set $\left\{\mathcal{A}_{i}\right\}$ or by the set $\left\{\gamma_{i}\right\}$. The relations (6.3) between these conjugate variables $\left\{\mathcal{A}_{i}\right\}$ and $\left\{\gamma_{i}\right\}$ are generated from the generalized thermodynamic potential $\Psi$ of eq.(6.4). The von Neumann entropy (6.3) of the state (6.1), when regarded as a function of the variables $\mathcal{A}_{i}$, appears as the Legendre transform of $\Psi\left(\left\{\gamma_{i}\right\}\right)$. We denote it as

$$
S_{R}\left(\left\{\mathcal{A}_{i}\right\}\right)=S\left(D_{0}\right)=\Psi+\sum_{i} \gamma_{i} \mathcal{A}_{i}
$$

and it alternatively generates the relations between the conjugate sets $\left\{\mathcal{A}_{i}\right\}$ and $\left\{\gamma_{i}\right\}$ through 


$$
\frac{\partial S_{R}}{\partial \mathcal{A}_{i}}=\gamma_{i}
$$

We term $S_{R}$ the relevant entropy associated with the set $R \equiv\left\{A_{i}\right\}$ of relevant observables. By construction it is the maximum value. $S\left(D_{0}\right)$ of the uncertainty - as measured by the von Neumann entropy $S(D)$ — of the various microscopic states $D$ which are equivalent as regards the expectation values

$$
\left\langle A_{i}\right\rangle=\operatorname{Tr} A_{i} D=\operatorname{Tr} A_{i} D_{0}=\mathcal{A}_{i}
$$

of the relevant observables, but which are inequivalent as regards the irrelevant observables (those which lie outside $R$ ). The relevant entropy thus characterizes the randomness of an incomplete statistical description of the system, involving only the set $\mathcal{A}_{i}$ at the considered time: it measures the amount of information which is missing when only the quantities $\left\langle A_{i}\right\rangle=\mathcal{A}_{i}$ are specified. While $D_{0}$ carries no other information than these expectation values, the other density operators $D$ satisfying (6.9) carry an extra amount of information which pertains to irrelevant observables and which is measured by

$$
S_{R}\left(\left\{\mathcal{A}_{i}\right\}\right)-S(D)
$$

The relevant entropy $S_{R}$ decreases as the set $R$ is enlarged. Consider a statistical ensemble of systems all prepared in the same manner, by controlling some set $R_{n}$ of observables. Imagine that we have no information at the start and that we determine better and better the state by learning the expectation values $\mathcal{A}_{i}$ for nested, larger and larger sets $R_{1} \subset R_{2} \subset \ldots \subset R_{n}$. (Or imagine that conversely we begin with the full statistical knowledge of $R_{n}$ and discard information by steps, until we keep only information on $R_{1}$.) Each step corresponds to a positive gain (or loss) of information. This is expressed by the inequalities

$$
S_{R_{1}}>S_{R_{2}}>\ldots>S_{R_{n}}
$$

for the relevant entropies associated with each set.

An exercise on relevant entropies can be found in ref. [1], exerc. 3c.

\section{Reduction of the description}

The approach of $\S 6$ covers the foundations of thermostatics. New inference problems arise, however, in non-equilibrium statistical mechanics. 
We first have to assign a density operator $D\left(t_{0}\right)$ to the system at the initial time $t_{0}$. Some macroscopic variables $\mathcal{A}_{i}$ are controlled externally so as to prepare the initial state, and this preparation is of course incomplete. We wish to infer therefrom the complete statistical description which is required to work out the microscopic approach of statistical physics. Such a question has been solved in $\S 6$, where we showed that the initial state $D\left(t_{0}\right)$ should have the form (6.1) in terms of the observables $A_{i}$ under experimental control. Actually these observables which can be handled in practice to prepare the system may constitute only a subset of the chosen set $R$ of relevant observables. In spite of that, we note that $D\left(t_{0}\right)=D_{0}\left(t_{0}\right)$ is still expressed as (6.1) in terms of the full set $R$, provided we associate a vanishing multiplier $\gamma_{j}\left(t_{0}\right)=0$ with any relevant observable $A_{j}$ that is not controlled in the preparation. The values of the multipliers $\gamma_{i}\left(t_{0}\right)$ associated with the controlled data $\mathcal{A}_{i}\left(t_{0}\right)$ are still determined by (6.5), while we infer from these controlled data the expectation values of the remaining, non controlled relevant observables by means of

$$
A_{j}\left(t_{0}\right)=-\left.\frac{\partial \Psi}{\partial \gamma_{j}}\right|_{\gamma_{j}=0} .
$$

The next problem is to make reasonable statistical predictions about any observable $B$ at any time $t$ subsequent to $t_{0}$ (or symmetrically to make retrodictions for times $t$ earlier than $\left.t_{0}\right)$. Starting from the initial condition $D\left(t_{0}\right)=D_{0}\left(t_{0}\right)$ inferred above from the data $\mathcal{A}_{i}\left(t_{0}\right)$, we determine $D(t)$ (at least in principle if not in practice) by solving the Liouville-von Neumann equation (1.2). The required quantities are then obtained as

$$
\langle B\rangle_{t}=\operatorname{Tr} B D(t) .
$$

The equation of motion (1.2) for $D(t)$ entails the constancy of the von Neumann entropy (3.2), which keeps its initial value:

$$
S[D(t)]=S_{R}\left[\left\{\mathcal{A}_{i}\left(t_{0}\right)\right\}\right] .
$$

This equation expresses that the complete statistical description provided by $D(t)$ conserves the information that was initially stored in the system through the controlled data $\mathcal{A}_{i}\left(t_{0}\right)$. This conservation is natural, owing to the reversibility of the microscopic evolution. However, $D(t)$ has no reason to keep the exponential form (6.1) that it had at the time $t_{0}$. Hence, with respect to the relevant set $R, D(t)$ is generally biased: the information that it carries pertains not only to this set $R$ (as at the initial time $t=t_{0}$ ) but also to irrelevant observables. The evolution thus generates some transfer of information from relevant to irrelevant variables. 
Let us make this point clearer by getting rid of the irrelevant information at each time. We thus wish to study the relevant quantities.

$$
\mathcal{A}_{i}(t)=\left\langle A_{i}\right\rangle_{t}=\operatorname{Tr} A_{i} D(t)
$$

irrespective of the other variables. The method of $\S 6$ allows us to discard the information carried by the latter, irrelevant variables. It provides us with a state $D_{0}(t)$, which accounts for the values $\left\langle A_{i}\right\rangle_{t}$ given by (7.4) for the relevant quantities and which, contrary to $D(t)$, is unbiased with respect to the set $R$. The values of $\langle B\rangle_{t}$ provided by $D_{0}(t)$ are those inferred from the knowledge of the set $\mathcal{A}_{i}(t)$ only; they differ from the actual values (7.2) which are inferred from the initial conditions $\mathcal{A}_{i}\left(t_{0}\right)$ and from the microscopic evolution. The missing information associated with the sole knowledge of the set (7.4) is the relevant entropy $S_{R}\left[\left\langle\mathcal{A}_{i}(t)\right\rangle\right]$, i.e., the von Neumann entropy $S\left[D_{0}(t)\right]$ of the unbiased state $D_{0}(t)$.

We are thus led to introduce, beside the complete statistical microscopic description through $D(t)$, a reduced description in terms of a simpler density operator $D_{0}(t)$ which carries the sole information on the relevant set $R$. At each time, we associate with $D(t)$ a reduced state $D_{0}(t)$ which has the exponential form (6.1) and which satisfies the conditions

$$
\operatorname{Tr} A_{i} D_{0}(t)=\operatorname{Tr} A_{i} D(t)=\mathcal{A}_{i}(t) .
$$

The reduced state $D_{0}(t)$ accompanies $D(t)$ in its motion. The parameters $\gamma_{i}(t)$ of (6.1) which determine $D_{0}(t)$ are obtained from (7.5). The correspondence between the sets $\left\{\gamma_{i}(t)\right\}$ and $\left\{\mathcal{A}_{i}(t)\right\}$ is implemented by (6.5) or (6.8), so that we can characterize the reduced state equivalently by the set $\left\{\mathcal{A}_{i}(t)\right\}$, by the set $\left\{\gamma_{i}(t)\right\}$ or by the density operator $D_{0}(t)$.

The information carried by this reduced description is smaller than that carried by the full description through $D(t)$. The difference

$$
S\left[D_{0}(t)\right]-S[D(t)]=S_{R}\left[\left\{\mathcal{A}_{i}(t)\right\}\right]-S_{R}\left[\left\{\mathcal{A}_{i}\left(t_{0}\right)\right\}\right],
$$

where we used (7.3), measures the irreversibility with respect to the set $R$. It is the amount of information which has leaked from the relevant to the irrelevant set. It is also interpreted as the transfer of disorder from the irrelevant to the relevant set. According to (7.3), the total disorder $S[D(t)]$ remains constant, while (7.6) shows that the disorder associated with the relevant set has increased between the times $t_{0}$ and $t$.

Altogether, we have been able to relate the macroscopic description in terms of the sole variables $\mathcal{A}_{i}(t)$ to the microscopic, detailed description of statistical mechanics. The following scheme was brought out: 


$$
\left\{\mathcal{A}_{i}\left(t_{0}\right)\right\} \Rightarrow D\left(t_{0}\right) \leftrightarrow D(t) \mapsto\left\{\mathcal{A}_{i}(t)\right\} \Rightarrow D_{0}(t) .
$$

From the data $\left\{\mathcal{A}_{i}\left(t_{0}\right)\right\}$ which are controlled at the initial time, we first inferred the state $D\left(t_{0}\right)=D_{0}\left(t_{0}\right)$ at that time by means of the maximum entropy criterion. The solution of the Liouville-von Neumann equation, which is the basic dynamical equation in statistical mechanics, related $D(t)$ to $D\left(t_{0}\right)$ reversibly. We then found the relevant quantities $\left\{\mathcal{A}_{i}(t)\right\}$ through (7.4). Eventually we inferred $D_{0}(t)$ from the latter set, using again the maximum entropy criterion. This last step will be used in $\S 8$ to eliminate explicitly the irrelevant variables. The introduction of $D_{0}(t)$ has also been necessary to define the relevant entropy, which according to (7.6) characterizes the loss of information in the step $D(t) \mapsto$ $\left\{\mathcal{A}_{i}(t)\right\}$ or the irreversibility of the correspondence from $\left\{\mathcal{A}_{i}\left(t_{0}\right)\right\}$ to $\left\{\mathcal{A}_{i}(t)\right\}$.

\section{The projection method}

Macroscopic descriptions of dynamical processes rely on more or less phenomenological equations for the macroscopic variables $\left\{\mathcal{A}_{i}(t)\right\}$. We now consider a major problem of statistical physics, namely justifying these macroscopic equations from the microscopic, more fundamental theory. To this is aim, we have to get rid of the irrelevant microscopic variables so as to work out the scheme (7.7) and to find equations of motions for the set $\left\{\mathcal{A}_{i}(t)\right\}$ only. The projection method of Nakajima and Zwanzig (4.5) achieves this goal, at least formally but quite generally. We sketch it here in order to shed light on the meaning of varied macroscopic dynamical equations.

We first note that the knowledge of the time-dependence of the set $\left\{\mathcal{A}_{i}(t)\right\}$ is equivalent to that of the reduced state $D_{0}(t)$. Indeed, we can deduce $\mathcal{A}_{i}$ from $D_{0}$ through (7.5). Conversely $D_{0}$, as expressed by (6.1), (6.3), depends on time through the variables $\left\{\gamma_{i}\right\}$, which in turn are related to the variables $\left\{\mathcal{A}_{i}\right\}$ by (6.5) or (6.8). We shall in the following regard $D_{0}$ as a function of the set $\left\{\mathcal{A}_{i}\right\}$, and as a function of time through this set.

The time-dependence of the reduced state $D_{0}$ that we thus seek for should be obtained from the Liouville-von Neumann equation of motion for the full $D$. We shall work out this derivation in a Liouville representation ( $\$ 2)$, where both the density operators and the observables are regarded as vectors in two conjugate vector spaces. In such a representation the latter equation of motion as well as the correspondence from $D$ to $D_{0}$ will be expressed by formally simple equations. On the one hand the Liouville-von Neumann equation is written as (2.3) in terms of the liouvillian superoperator $\mathrm{L}$.

On the other hand, $D_{0}$, which is a function of the set $\left\{\mathcal{A}_{i}\right\}$ through (6.1), (6.3) and (6.8), follows from $D$ through the evaluation of the set $\left\{\mathcal{A}_{i}\right\}$ by means of (7.4). It can be shown that this construction amounts, in a Liouville representation 
where $D$ and $D_{0}$ are regarded as vectors in the Liouville space, to a projection of $D$ onto the surface (6.1):

$$
D_{0}=\mathrm{P} D
$$

The maximum entropy criterion is thus implemented as a projection. In a Hilbert space representation, the projection superoperator $\mathrm{P}$ is a tensor with $2 \times 2$ indices, which acts in the same way (2.4) as the liouvillian on the density matrix $D$. The projection $\mathrm{P}$ depends on the variables $\left\{\mathcal{A}_{i}\right\}$. It acts on its left hand side upon the observables, and satisfies in particular

$$
A_{i} \mathrm{P}=A_{i}, I \mathrm{P}=I
$$

The projection thus keeps the relevant set $R$ of observables invariant. These facts are easily checked from the expression of $\mathrm{P}$,

$$
\mathrm{P}=D_{0} \otimes I+\sum_{i} \frac{\partial D_{0}}{\partial \mathcal{A}_{i}} \otimes\left(A_{i}-\mathcal{A}_{i} I\right)
$$

which however we shall not need below. Eq. (8.3) exhibits P as a sum of elementary projections, each of which is the tensor product of a state-like vector and an observable-like vector, that lie in the conjugate Liouville spaces; the partial derivative $\partial D_{0} / \partial \mathcal{A}_{i}$ is evaluated by regarding $D_{0}$ as a function of the variables $\left\{\mathcal{A}_{i}\right\}$.

The complementary projection superoperator $\mathrm{Q}$ onto the irrelevant space is defined as

$$
\mathrm{Q}=\mathrm{I}-\mathrm{P}
$$

where I is the unit superoperator in the Liouville space. The properties $\mathrm{P}^{2}=$ $P, Q^{2}=Q$ and $P Q=0$ show that the Liouville formalism helps separating the relevant and the irrelevant components. The density operator $D$ can thereby be split at each time into its reduced relevant part $D_{0}$ and its irrelevant part $D_{1}$ defined by

$$
D_{1}=\mathrm{Q} D
$$

We wish to find the time-dependence of $D_{0}$ from the Liouville-von Neumann equation (2.3) which deals with the full $D$. We take therefore the time-derivative of (8.1), using (2.3). This yields 


$$
\frac{d D_{0}}{d t}=\frac{d \mathrm{P}}{d t} D_{0}+\mathrm{PL} D_{0}+\mathrm{PL} D_{1}
$$

where the time-dependence of $\mathrm{P}$ occurs through the variables $\left\{\mathcal{A}_{i}\right\}$. Apart from its last term where $D_{1}$ is as get unknown, (8.6) is a differential equation for $D_{0}$ or for the set $\left\{\mathcal{A}_{i}\right\}$. It can be written equivalently, using (7.5), as the set

$$
\frac{d \mathcal{A}_{i}}{d t}=\left(A_{i} ; \mathrm{L} D_{0}\right)+\left(A_{i} ; \mathrm{L} D_{1}\right)
$$

where we made use of the notation (2.2) for a scalar product in the Liouville space and where we took (8.2) into account.

In order to eliminate $D_{1}$ from (8.7), we write the equation of motion for $D_{1}$, by taking the time-derivation of (8.5), which yields

$$
\frac{d D_{1}}{d t}+\frac{d \mathrm{P}}{d t} D_{1}-\mathrm{QL} D_{1}=\mathrm{QL} D_{0}
$$

The equations (8.6) and (8.8) couple the projected parts $D_{0}$ and $D_{1}$ of $D$. As (8.8) is linear in $D_{1}$, with coefficients depending on $D_{0}$, it can be solved formally by introducing its Green's function $\mathrm{W}\left(t, t^{\prime}\right)$. This quantity is a superoperator, which is the solution of

$$
\left(\frac{\partial}{\partial t}+\frac{d \mathrm{P}}{d t}-\mathrm{QL}\right) \mathrm{W}\left(t, t^{\prime}\right)=\mathrm{Q} \delta\left(t-t^{\prime}\right)
$$

with $\mathbf{W}\left(t, t^{\prime}\right)=0$ for $t<t^{\prime}$. Using the initial condition $D_{1}\left(t_{0}\right)=0$ that results from $D\left(t_{0}\right)=D_{0}\left(t_{0}\right)(\S 7)$, we thus express $D_{1}$ in terms of $D_{0}$ as

$$
D_{1}(t)=\int_{t_{0}}^{t} d t^{\prime} \mathrm{W}\left(t, t^{\prime}\right) \mathrm{L} D_{0}\left(t^{\prime}\right)
$$

Insertion of (8.10) into (8.7) achieves one goal, finding a set of dynamical equations for the variables $\mathcal{A}_{i}(t)$ alone:

$$
\frac{d \mathcal{A}_{i}}{d t}=\left(\mathcal{A}_{i} ; \mathrm{L} D_{0}(t)\right)+\left(\mathcal{A}_{i} ; \int_{t_{0}}^{t} d t^{\prime} \mathrm{LW}\left(t, t^{\prime}\right) \mathrm{L} D_{0}\left(t^{\prime}\right)\right)
$$

where $\mathrm{W}$ was defined by (8.9). The elimination of the irrelevant variables has resulted in a set of integro-differential equations which are non-linear and nonmarkovian. Indeed the last of (8.11) does not depend on $D_{0}(t)$ alone as does the first term, but on $D_{0}$ at earlier times, directly through $D_{0}\left(t^{\prime}\right)$ and indirectly 
through the projections $\mathrm{P}$ and $\mathrm{Q}$ which enter the definition (8.9) of $\mathrm{W}$. The evolution of the set $\left\{\mathcal{A}_{i}\right\}$ thus involves a memory, which is characterized by the memory kernel $\mathrm{W}\left(t, t^{\prime}\right)$. The latter superoperator depends on the history of $\mathcal{A}_{i}$ between the times $t^{\prime}$ and $t$.

This first term in (8.11) represents the direct coupling of the observables within the relevant set $R$; it involves the components PLP of the liouvillian. The last, retarded term is interpreted as the effect of an indirect coupling through the irrelevant observables. Since W satisfies

$$
\mathbf{W}\left(t, t^{\prime}\right)=\mathrm{Q}(t) \mathbf{W}\left(t, t^{\prime}\right) \mathbf{Q}\left(t^{\prime}\right)
$$

and since its definition (8.9) involves only the components QLQ of the liouvillian, it describes the time-evolution of the irrelevant set of variables that we have eliminated. This irrelevant set is coupled in (8.11) to the set $R$ of interest, at the time $t$ through the cross components PLQ of the liouvillian, at the earlier times $t^{\prime}$ through its components QLP.

We noted that the von Neumann entropy $S(D)$, associated through (3.2) with the complete statistical description $D$, remains constant during the evolution generated by (1.2), expressing that this evolution conserves information or disorder. We have here to associate with the reduced equation of motion (8.11) the relevant entropy $S_{R}\left(\left\{\mathcal{A}_{i}\right\}\right)$ expressed by (6.7), which characterizes the disorder in the relevant set $R$. Its time variation, obtained from (6.8) and (8.11) as

$$
\begin{aligned}
\frac{d S_{R}}{d t} & =\sum_{i} \gamma_{i} \frac{d \mathcal{A}_{i}}{d t} \\
& =\left(-\ln D_{0}(t) ; \int_{t_{0}}^{t} d t^{\prime} \operatorname{LW}\left(t, t^{\prime}\right) \mathrm{L} D_{0}\left(t^{\prime}\right)\right),(8.13)
\end{aligned}
$$

has no reason to vanish. In fact, the positivity of (7.6) shows that, at least between the times $t_{0}$ and $t$, the relevant entropy increases. The expression (8.13), after integration over time, relates this loss of information to the coupling of the relevant set $R$ with the irrelevant observables.

\section{Short-memory approximation}

Our whole construction relied on the choice of the relevant set $R$, which guided by macroscopic physics, itself based on experiment and on phenomenological theory. The macroscopic dynamical equations for the variables $\mathcal{A}_{i}(t)$ usually have the form of differential equations. In contrast, the exact equations (8.11) have a different structure, involving memory effects for whatever choice of $R$. A further 
step is thus needed to found the macroscopic equations upon the microscopic theory.

We wish the last term of (8.11) to depend approximately only on the set $\left\{\mathcal{A}_{i}(t)\right\}$ at the considered time $t$, not at earlier times. This can be achieved if the choice of the relevant set $R$ satisfies some conditions about time-scales. Actually, the main contribution to the integral over $t^{\prime}$ in (8.11) should arise from times $t^{\prime}$ sufficiently close to the upper bound $t$ so that $D_{0}(t)$ does not differ significantly from $D_{0}(t)$. This requires the weight

$$
\mathrm{P}(t) \operatorname{LW}\left(t, t^{\prime}\right) \operatorname{LP}\left(t^{\prime}\right)
$$

which multiplies $D_{0}\left(t^{\prime}\right)$ and which accounts for the dynamics of the irrelevant variables, to decrease sufficiently fast as $t-t^{\prime}$ increases. The relevant set should thus have been chosen by selecting the slowest variables. We understand the possibility of a short time-range $\tau$ for the expression (9.1) by noting that it is equal to $\mathrm{P}(t) \operatorname{LQ}(t) \operatorname{LP}(t)$ when $t=t^{\prime}$, and that for $t>t^{\prime}$ it includes a sum of a large number of terms, associated with the evolution of the irrelevant degrees of freedom which oscillate rapidly and interfere destructively as $t-t^{\prime}$ increases. If (9.1) is thus negligible beyond the delay $\tau$, the memory in the evolution of the relevant set due to their coupling with the irrelevant ones is lost after the time-lapse $\tau$.

We can then replace $D_{0}\left(t^{\prime}\right)$ by $D_{0}(t)$ in the equations (8.11), which become approximately

$$
\frac{d \mathcal{A}_{i}}{d t} \simeq\left(A_{i} ;[\mathrm{L}+\mathrm{LK}(t) \mathrm{L}] D_{0}(t)\right)
$$

we defined $\mathbf{K}(t)$ as

$$
\mathrm{K}(t)=\int_{t_{0}}^{t} d t^{\prime} \mathrm{W}\left(t, t^{\prime}\right)
$$

The lower bound $t_{0}$ in (9.2) is not significant since the integrand is negligible outside the interval $t>t^{\prime} \gtrsim t-\tau$. The superoperator $\mathrm{K}$ acts only on the irrelevant space since $\mathrm{K}(t)=\mathrm{Q}(t) \mathrm{K}(t) \mathbf{Q}(t)$, as seen from (8.12) and from the slow variation of $\mathrm{Q}(t)$ on the scale $\tau$. The bracket in (9.2) can equivalently be replaced by $\mathrm{P}[\mathrm{L}+\mathrm{LKL}] \mathrm{P}$, which shows that it acts on the relevant space only.

The projection method, completed by this short-memory approximation, has thus led to the equations of motion (9.2) which provide a microscopic basis for the macroscopic dynamics. The possibility of describing the system by differential equations for some partial set of relevant variables, without considering the remaining irrelevant microscopic variables, relied on the recognition of different 
characteristic time-scales for the two sets. The influence on the macroscopic dynamics of the irrelevant variables which have been eliminated then occurs through the factor PLKLP in (9.2). The method is general and flexible, as examples will show. It is however formal, since the solution of the equation (8.9) for $\mathrm{W}$ is at least as difficult as that of the Liouville-von Neumann equation. Further approximation (such as perturbative expansions) specific to the considered problem, are therefore needed to evaluate $\mathrm{K}$ from first principles. A rough approximation consists in estimating only the memory-time $\tau$ and in taking $\mathrm{K} \simeq \tau \mathrm{Q}$.

When the approximation (9.2) is valid, the dissipation (8.13) has the instantaneous form

$$
\frac{d S_{R}}{d t}=\left(-\ln D_{0} ; \mathrm{LKL} D_{0}\right)
$$

It is positive at each time, expressing a continuous flow of disorder from the irrelevant to the relevant variables. Although dissipation appears from (8.13) to be a memory effect, it is in fact a short-memory effect, taking place on the time-scale $\tau$. The reconciliation of microscopic reversibility with macroscopic irreversibility thus requires the existence of two different time-scales.

\section{The thermodynamic entropy}

We have seen in $\S 5$ that the macroscopic state of a system evolving in a thermodynamic regime is characterized by a set of local variables $\mathcal{A}_{i a}$ associated, for each subsystem located at $a$ with some or other quantity $i$. These quantities may flow from a subsystem $a$ to another, and thermodynamics deals with such a transport [8]: thermal conduction corresponds to flow of energy, diffusion or electric current to flow of particles, fluid dynamics to flow of momentum. The thermodynamic equations provide the time-dependence of the variables $\mathcal{A}_{i a}$. They can be decomposed into: (i) Conservation laws relating the time-derivatives $d \mathcal{A}_{i a} / d t$ to fluxes from each subsystem to its neighbours. (ii) For each subsystem $a$, relations between the variables $\mathcal{A}_{i a}$ and their conjugate local intensive variables $\gamma_{j a}$, such as temperature, chemical potential or velocity; these relations are at each point and each time the same as in thermostatics. (iii) Definition of affinities as differences $\gamma_{i a}-\gamma_{i b}$ or as gradients of the intensive variables. (iv) Responses of the fluxes to the affinities.

The equations for the set $\left\{\mathcal{A}_{i a}\right\}$ characterize the exchanges which occur between the subsystems. Such exchanges take place on a time-scale which is large compared the time-scale $\tau$ of the microscopic processes. In the present case, the latter time $\tau$ corresponds to the delay after which the effect of the perturbation of a microscopic variable has been forgotten. It is thus the relaxation time which brings the system to local equilibrium. Thereafter the evolution slows down, and 
proceeds in the thermodynamic or hydrodynamic regime where the dynamics involves the local quantities $\mathcal{A}_{i a}$ only. In a gas, $\tau$ is of the order of the delay between two successive collisions of a particle.

The occurence of a larger time-scale for the thermodynamic variables $\mathcal{A}_{i a}$ is related to the fact that these variables are conserved or nearly conserved. Indeed, the conservation laws imply that $\mathcal{A}_{i a}$ can increase only if $\mathcal{A}_{i b}$ in a neighbouring subsystem $b$ decreases, under the effect of a flux from $b$ to $a$. This requires a coupling between the subsystems $a$ and $b$. However, due to the macroscopic size of the subsystems, such a coupling is an interface effect which may be relatively weak; this hinders the fluxes and hence the time-variation of the variables $\mathcal{A}_{i a}$.

The microscopic incomplete description associated with the set of thermodynamic variables $\mathcal{A}_{i a}$ is based on a reduced density operator $D_{0}$ of the form (6.1). Separation of the observables $\mathcal{A}_{i a}$ according to the subsystem $a$ that they refer to expresses $D_{0}$ as a tensor product

$$
D_{0}=\prod_{a} D_{a}, D_{a}=\exp \left[-\Psi_{a}-\sum_{i} \gamma_{i a} A_{i a}\right]
$$

of elementary density operators $D_{a}$, each of which refers to one of the subsystems. In fact $D_{a}$ has exactly the same form as the density operator of the system $a$ alone in thermostatic equilibrium. For each subsystem the relations between the variables $\mathcal{A}_{i a}$ and $\gamma_{j a}$ are thus the same as in thermostatics. We can therefore identify the multipliers $\gamma_{i a}$ of (10.1) with the corresponding intensive variables of thermodynamics. We can also identify the set (9.2) with the thermodynamical equations of motion.

Moreover the factorization (10.1) of $D_{0}$ implies that the relevant entropy (6.7) associated with the thermodynamic variables is simply the sum

$$
S_{R}\left(\left\{\mathcal{A}_{i a}\right\}\right)=\sum_{a} S\left(D_{a}\right)
$$

of the von Neumann entropies $S\left(D_{a}\right)$, each of which is equal to the thermostatic entropy of the subsystem $a$. This allows us to identify the relevant entropy $S_{R}\left(\left\{\mathcal{A}_{i a}\right\}\right)$ relative to the thermodynamic variables with the entropy $S_{\text {th }}$ of thermodynamics.

The Second Law of thermostatics refers to a system in local equilibrium at the initial time $t_{0}$, in global equilibrium at a final time $t_{1}$. In the interval, the evolution does not need to take place in the thermodynamic regime. We may for instance deal with an explosive chemical reaction, or a shock wave, and the shortmemory approximation of $\S 9$ is thus not necessarily valid. However, while the von Neumann entropy $S[D(t)]$ remains constant, the positivity of (7.6) implies 
that $S_{R}\left[\left\{\mathcal{A}_{i a}\left(t_{1}\right)\right\}\right]>S_{R}\left[\left\{\mathcal{A}_{i a}\left(t_{0}\right)\right\}\right]$, which proves microscopically the Second Law.

If the evolution takes place in the thermodynamic regime according to (9.2), the dissipation (9.4) is at each time positive, which means that $S_{\text {th }}$ keeps increasing. This property is the dynamical form of the Second Law, expressed by the Clausius-Duhem inequality

$$
\frac{d S_{\mathrm{th}}}{d t} \geqslant 0
$$

\section{The Boltzmann entropy}

We turn to the Boltzmann description of a gas $(\S 5)$. Here the relevant observables $A_{i}$ are the single-particle random variables; their expectation values are the set $\left\{\mathcal{A}_{i}\right\}=f(\mathbf{r}, \mathbf{p})$, i.e., the density of particles in the single-particle phase space. This description is more detailed than the thermodynamic description of the gas, which would rely only on the densities of particles, of energy and of momentum in the ordinary space $\mathbf{r}$. It applies to more general regimes than the thermodynamic regime. In particular, in the ballistic regime, the time between collisions which characterizes the thermalization is larger than the time during which $f(\mathbf{r}, \mathbf{p})$ changes significantly; the mean free path is larger than the distance over which $f$ changes. No local temperature, no hydrodynamic flow can then be defined.

While $f(\mathbf{r}, \mathbf{p})$ plays the rôle of the macroscopic variables $\left\{\mathcal{A}_{i}\right\}$, the irrelevant variables are here the correlations between the particles of the gas. Such correlations are created by collisions; the characteristic time associated with their dynamics is the duration of a collision. Over time-scales larger than this very brief duration, and over distances larger than the range of the interactions, the dynamics of $f(\mathbf{r}, \mathbf{p} ; t)$ is governed by the semi-phenomenological Boltzmann equation

$$
\frac{\partial f(\mathbf{r}, \mathbf{p} ; t)}{\partial t}=-\frac{\mathbf{p}}{m} \cdot \nabla_{r} f+\mathcal{I}[f]
$$

This equation of motion for a gas is adequate under conditions much more general than the equations of thermodynamics. Like the latter ones it should be supplemented by boundary conditions accounting for the vessel in which the gas is enclosed. We shall consider it as a macroscopic equation and $f(\mathbf{r}, \mathbf{p})$ as a continuous set of macroscopic variables, whereas the Liouville equation for the full $D$ is the exact microscopic equation. The last term $\mathcal{I}[f]$ is the collision integral, a quadratic functional of $f$ involving integration over momenta.

By introducing the quantity 


$$
H(t) \equiv \int d^{3} \mathbf{r} d^{3} \mathbf{p} f \ln f
$$

Boltzmann proved that his equation (11.1) satisfies the H-theorem, namely

$$
\frac{d H}{d t} \leqslant 0
$$

This exhibits the irreversibility of the Boltzmann equation, which contrasts with the reversibility of the more basic Liouville equation.

At the microscopic level the Boltzmann description relies on a reduced density $D_{0}$ in the $6 N$-dimensional phase space, which is the classical equivalent of (6.1). The random variables $A_{i}$ entering it are the classical single-particle quantities, so that $D_{0}$ describes an uncorrelated state. The projection (8.1) amounts here to chop off the correlations from $D$, while keeping the expectation values $f(\mathbf{r}, \mathbf{p})$ of single-particle quantities invariant. The Boltzmann equation is then identified with the result (9.2) of the projection method, the short-memory approximation being here justified by the short duration of the collisions. The two terms of (11.1) actually correspond to the two terms of (9.2).

The relevant entropy associated with the Boltzmann variables $f(\mathbf{r}, \mathbf{p})$ is found by taking the classical limit of $(6.4),(6.5),(6.7)$, where the trace is replaced by the measure (2.6). We obtain

$$
S_{\mathrm{B}} \equiv S_{R}(f)=\int d^{3} \mathbf{r} d^{3} \mathbf{p} f(\mathbf{r}, \mathbf{p})\left[1-\ln h^{3} f(\mathbf{r}, \mathbf{p})\right]
$$

This expression defines the Boltzmann entropy $S_{\mathrm{B}}$, which is relevant for the dynamics of a gas in whatever regime, provided the range of the interparticle potential is short compared to the distances between particles. Since the integral of $f$ is the particle number, the Boltzmann entropy $S_{\mathrm{B}}$ is proportional to Boltzmann's $H$, within the sign and within an additive constant. Thus the $H$-theorem expresses merely the increase (9.4) of the Boltzmann entropy.

The $H$-theorem should not be confused with the Second Law. On the one hand, it applies only to gases. On the other hand, it covers much more general situations, since in thermostatics the Second Law requires local equilibrium for the initial state, global equilibrium for the final state, and since in thermodynamics the Clausius-Duhem inequality requires local equilibrium at all times.

The thermodynamics of gases is recovered from the Boltzmann description in regimes for which $f$ varies slowly in space on the scale of the mean free path, slowly in time on the scale of the delay between collisions. In this limit, it can be shown that of $f(\mathbf{r}, \mathbf{p}, t)$ does not differ much from a single-particle density in phase space having in terms of $\mathbf{p}$ the maxwellian form 


$$
f_{0}(\mathbf{r}, \mathbf{p}, t)=\exp \left[-\gamma_{1}-\gamma_{2} \frac{p^{2}}{2 m}-\gamma_{3} \cdot \mathbf{p}\right],
$$

where $\gamma_{1}, \gamma_{2}$ and $\gamma_{3}$ depend on $\mathbf{r}$ and $t$. The latter quantities are interpreted as the local intensive variables in the local equilibrium regime of thermodynamics, for instance $1 / k \gamma_{2}$ as the local temperature (the energy of the gas includes practically the kinetic energy only of the particles). The Boltzmann entropy (11.4) reduces to the thermodynamic entropy $S_{\mathrm{th}}$, the $H$-theorem to the thermodynamic dissipation (10.3). The thermodynamic equations of motion can also be obtained from the Boltzmann equation (11.1). The Chapman-Enskog method, on which this derivation is based, appears as an application of the projection method. Here $f$ is regarded as the set of microscopic variables. We thus replace in the general formalism $D$ by $f$, and the Liouville-von Neumann equation by the Boltzmann equation (see for instance [1], chap. 15). Like $D$ in eqs. (8.1) and (8.5), $f$ is split into $f=f_{0}+f_{1}$, where $f_{0}$, of the form (11.5), is obtained from $f$ by means of a projection in the space of the functions of $\mathbf{p}$. The elimination of $f_{1}$ is made here feasible by regarding $f_{1}$ as small compared to $f_{0}$ in the considered regime, and it results in the Navier-Stokes and Fourier equations.

\section{Conclusion: the multiplicity of entropies}

The above example of a gas exhibits the occurrence in non-equilibrium statistical mechanics of several different entropies for a single system.

(i) The von Neumann entropy $S(D)$ defined by (3.2) is associated with the complete statistical description of the gas. It accounts in particular for the order carried by the correlations between its particles, which develop as collisions take place, even if they are absent at the initial time. This entropy remains constant, expressing the reversibility of the microscopic evolution and the conservation of the initial information.

(ii) The Boltzmann entropy $S_{\mathrm{B}}$ defined by (11.4) is the relevant entropy associated with the single-particle properties only, in an incomplete description disregarding all the correlations. Its increase in time, the $H$-theorem, expresses the irreversible loss of information, or of order, which take place from the singleparticle quantities towards the correlations. Actually, although the interactions an essential in the dynamics of the gas, the correlations which may exist between a particle and other ones are not effective when this particle undergoes a new collision; this is Boltzmann's Stosszahlansatz, that he used to justify his equation (11.1).

(iii) The thermodynamic entropy $S_{\text {th }}$ defined by (10.2) is the relevant entropy associated with only the locally conserved densities of particles, of energy and of momentum at each point. In the thermodynamic regime where $f$ has the 
form (11.5), the short-memory approximation is valid and it ensures that $S_{\text {th }}$ continuously increases. This expresses the dissipation due to viscosity and to thermal conduction.

(iv) The thermostatic entropy $S_{\text {eq }}(\mathcal{N}, \mathcal{U})$ defined in $\S 6$ can also be regarded as the relevant entropy associated with the total particle number and the total energy. These quantities are the globally conserved ones; momentum is locally conserved but not globally due to the collisions onto the walls, it vanishes at equilibrium. This definition holds at any time for a gas off equilibrium, with $\mathcal{N}$ and $\mathcal{U}$ keeping their initial values. However $S_{\text {eq }}$, which does not vary, has no physical interest, except after the very long, global relaxation time has elapsed. The gas has then been brought to thermostatic equilibrium through the evolution generated by the Boltzmann equation.

Because the above four descriptions are more and more incomplete, their three relevant entropies satisfy the inequalitites (6.11), to wit,

$$
S_{\text {eq }}>S_{\text {th }}>S_{\text {B }}>S(D) .
$$

The entropies $S_{\text {th }}$ and $S_{\mathrm{B}}$ thus vary between the constant lower and upper bounds $S(D)$ and $S_{\text {eq }}$, and $S_{\mathrm{B}}$ never decreases. After local equilibrium is established, $S_{\mathrm{B}}$ and $S_{\text {th }}$ are nearly equal and both tend to $S_{\text {eq }}$ when the system reaches global equilibrium; as we just saw, $S_{\text {eq }}$ is of interest only afterwards. Likewise, $S_{\text {th }}$ has no interest before local equilibrium is reached, that is, during some time-lapse of the order of the delay between collisions. In this initial fast regime, the thermodynamic variables are inadequate. They obey only an impracticable equation of the type (8.11) including retardation effects, and not yet a thermodynamic equation of the type (9.2). Accordingly, nothing forces $d S_{\text {th }} / d t$ to be always positive at this stage of the evolution.

The contrast between the constancy of $S(D)$ and the increase of $S_{\mathrm{B}}$ is better understood by introducing still other relevant entropies [9]. Starting from the Boltzmann description based on single-particle quantities only, a hierarchy of more and more detailed descriptions are introduced by including two-particle correlations, then three-particle correlations, and so on. The sequence of associated relevant entropies $S_{2}, S_{3}, \ldots$ satisfies the inequalities

$$
S_{\mathrm{B}}>S_{2}>S_{3}>\cdots>S(D) .
$$

For an independent-particle initial state, all these entropies are equal at the initial time $t_{0}$. As $S_{\mathrm{B}}$ does, any $S_{n}$ begins to be equal to $S(D)$ before increasing so as to reach eventually $S_{\text {eq }}$ when global equilibrium is attained. The curves $S_{n}(t)$ are ordered according to (12.2). As $n$ increases, the rise of $S_{n}$ takes place later and later. This is necessary because, at any fixed time $t, S_{n}$ decreases with $n$ down to $S(D)$, which is attained when $n$ is so large that all the correlations created 
by the evolution from $t_{0}$ to $t$ are taken into account. For $t$ and $n$ both large, $S_{n}(t)$ behaves non-uniformly: it tends to $S_{\text {eq }}$ when $t \rightarrow \infty$ for fixed $t$. These two regimes correspond to $t \gg n \tau$ and to $t \ll n \tau$, respectively, where $\tau$ is the time between successive collisions, since each new collision adds an additional correlation to a cluster already correlated by the previous collisions.

Similar ideas apply to systems other than gases. In particular the adequacy of a partial description based on some choice of the set $R$ relies on the absence of significant memory effects in the resulting equations of motion (8.11). If such effects occur, there exist within the set that we regarded as irrelevant some observables which are coupled to those that we included in $R$ and which have a comparable characteristic time. A decrease of the relevant entropy indicates that information, or order, has been stored in such hidden variables, and is released later on towards the variables retained in our description. Any hidden variable of this type should therefore be included into the relevant set $R$, so as to ensure that the information flowing towards the discarded variables is irretrievably lost.

We illustrate this point by reconsidering the spin-echo experiment described in the end of $\S 3$. Let us first forget about the experimental possibility of rotating the spins by $\pi$ around the $x$-axis at the time $T$ by means of a pulse along this axis. The thermodynamics of spins is characterized by an entropy $S_{\mathrm{th}}(M)$ which is the relevant entropy associated with the macroscopic quantity, the magnetization M. It vanishes when all the spins are aligned, and increases as $M$ decreases. Thus the relaxation of $\mathbf{M}$, which can be described by a macroscopic equation of motion involving both rotation and damping, results as expected in the increase of $S_{\text {th }}$. However, the magnetic pulse at the time $T$ along the $x$-axis not only changes $\mathbf{M}$ but also changes the individual spins in a manner which keeps track of their history between the time 0 and the time $T$. Owing to this possibility of manipulation, the memory about the initial state is not lost at the time $T$ although the spins point in any direction of the plane. This apparent disorder of the spins reflects in fact the weak dispersion in the values at each site of the permanent field along $z$, since the spins have rotated between the time 0 and the time $T$ by an angle which is proportional to the local field. Due to this correlation between spins and fields, the total magnetization no longer follows between the times $T$ and $2 T$ the macroscopic relaxation equation, but an equation involving memory about the individual spin sites. The decrease between the times $T$ and $2 T$ of the entropy $S_{\text {th }}(M)$ down to its initial value zero reflects the possibility of retrieval of the initial information through this memory. We must thus include within the relevant set after the time $T$ the hidden variables, i.e., the expectation values of the individual spins instead of that of the total spin. This produces a set of differential equations without memory, valid at all times. The relevant entropy associated with the individual spins never decreases and is now meaningful, contrary to the thermodynamic entropy $S_{\text {th }}(M)$. 


\section{References}

[1] R. Balian, From microphysics to macrophysics: methods and applications of statistical physics, vol. I and II (Springer-Verlag, 1991, 1992). A second, revised edition is being prepared.

[2] N.L. Balazs and B.K. Jennings, "Wigner's function and other distribution functions in mock phase spaces", Phys. Reports 104 (1984) 347-391.

[3] M. Hillery, R.F. O'Connell, M.O. Scully and E.P. Wigner, "Distribution functions in physics: fundamentals" Phys. Reports 106 (1984) 121-167.

[4] R. Balian, Y. Alhassid and H. Reinhardt, "Dissipation in many-body systems: a geometric approach based on information theory", Phys. Reports 131 (1986) $1-146$.

[5] J. Rau and B. Müller, "From reversible quantum microdynamics to irreversible quantum transport", Phys. Reports 272 (1996) 1-59.

[6] E.T. Jaynes, "Information theory and statistical mechanics", Phys. Rev. 106 (1957) 620-630.

[7] R. Balian and N. Balazs, "Equiprobability, inference and entropy in quantum theory", Ann. Phys. 179 (1987) 97-144. This article makes use of various techniques drawn from quantum field theory, although it deal only with ordinary quantum mechanics. It may be used as an exercise in a course of quantum field theory.

[8] H.B. Callen, Thermodynamics and an introduction to thermostatistics (Wiley, 1985).

[9] J.E. Mayer and M.G. Mayer, Statistical mechanics, 2nd edition (Wiley, 1977) pp. $145-154$.

The above review articles contain extensive bibliographies to which the reader is referred. 